\title{
Influence of Glycidyl Methacrylate Grafted Multi-walled Carbon Nanotubes on Viscoelastic Behaviors of Polypropylene Nanocomposites
}

\author{
Young-Sun Shim and Soo-Jin Park^ \\ Department of Chemistry, Inha University, 253 Nam-gu, Incheon 402-751, Korea \\ ‘e-mail: sjpark@inha.ac.kr \\ (Received August 17, 2010; Accepted December 8, 2010)
}

\begin{abstract}
In this work, the effect of glycidyl methacrylate grafted multi-walled carbon nanotubes (GMA-MWCNTs) on the viscoelastic behaviors of polypropylene (PP) based nanocomposites was studied. The GMA-MWCNTs/PP was prepared using a bravender at $200^{\circ} \mathrm{C}$ by melt mixing as a function of GMA-MWCNT content. The viscoelastic behaviors of GMA-MWCNTs/PP nanocomposites were measured by a rheometer. It was found that the GMA-MWCNTs were homogeneously dispersed in the PP matrix. The GMA-MWCNTs/PP nanocomposites showed higher storage modulus, loss modulus, and shear viscosity compared to pure PP nanocomposites and the maximum value was shown at $2.0 \mathrm{wt} \%$ GMA-MWCNTs loading. These results were probably attributed to the strong interfacial interaction between the GMA-MWCNT and the PP matrix.
\end{abstract}

Keywords : Viscoelastic behaviors, Glycidyl methacrylate, Multi-walled carbon nanotubes

\section{Introduction}

Carbon nanotubes (CNTs) have generated great interest from both fundamental and practical points of view, and they have been proposed for many potential applications [1-4], such as sensors [5,6], field-emission devices [7,8], logic devices $[9,10]$, and probes in various chemistry and biology $[11,12]$. Especially, development of the polymer/CNTs composites has been widely studied because of its superior mechanical and electrical properties $[13,14]$. Several studies have been devoted to the uses of CNTs as fillers to improve performances of composites or to achieve new properties of the composites, due to the superior Young's modulus (up to $1 \mathrm{TPa}$ ) [13], large aspect ratio, and high electrical conductivity (up to $10^{3} \mathrm{~S} / \mathrm{cm}$ ) [14] of CNTs.

Among the most versatile polymer matrices, poly olefins, such as polypropylene (PP) are thermoplastics having a higher consumption because of their well-balanced physical and mechanical properties and their easy process ability at a relatively low cost. Mechanical properties of PP are generally modified by melt mixing with particulate (e.g. talc, mica, and clay) $[3,4]$ and fibrous (e.g. glass, jute, aramid, and carbon fibers) fillers [6-9] as well as by melt blending with other polymers [10-12]. Reinforcement at nanoscale to improve mechanical and other properties including changes in polymer crystallization behavior is being attempted [13].

However, the effective use of CNTs as fillers in polymer composites depends on the capacity to obtain a good dispersion of CNTs in the matrix, without damaging them or reducing their aspect ratio. Techniques such as end-group functionalization, use of ionic surfactants, shear mixing, and plasma coating have been used to improve dispersion and exfoliation of nanotubes in polymers. Polypropylene compatibility with fillers has been improved by matrix modification by grafting it with reactive moieties, such as acrylic acid, acrylic esters, and maleic anhydride [13]. Improvement in thermal and electrical properties of PP/CNT composites has been reported [14].

In this work, multi-walled carbon nanotubes were treated using glycidyl methacrylate by chemical modification and the effect of GMA-grafting on the MWCNT surfaces in the GMA-MWCNT/PP nanocomposites for viscoelastic behaviors was investigated.

\section{Experimental}

\subsection{Materials}

MWCNTs used in this study were synthesized by chemical vapor deposition and provided by Nanosolutions Co. The diameter was 10 25 nm with micrometer length, and the purity was $>95 \mathrm{wt} \%$. The MWCNTs were used in our experiment with further purification. Hydrochloric acid (35\%) was obtained from Burdick and Jackson. Hydrogenperoxide (38\%) from Sanchun chemical Co., Ltd. was used for efficient grafting of hydroxyl group. Glycidyl methacrylate (GMA, purity 97\%) and 3-Chlorotoluene (98\%) were purchased from Sigma-Aldrich. 


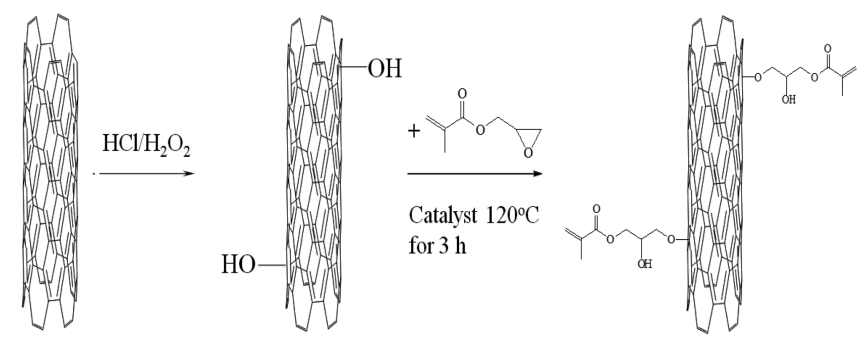

Fig. 1. Scheme of surface treatment for GMA-MWCNTs.

\subsection{Preparation of GMA-MWCNTs/PP nanocomposites}

The MWCNTs ( $1 \mathrm{~g})$ were pretreated with $\mathrm{HCl}(35 \%)$ at room temperature for $5 \mathrm{~h}$. And then, MWCNTs were added in $\mathrm{H}_{2} \mathrm{O}_{2}$ $(38 \%)$ and stirred about $12 \mathrm{~h}$ at $80^{\circ} \mathrm{C}$. Acid-modified MWCNTs were washed with distilled water for neutralization. After vacuum filtration with micro-sized polycarbonate membrane, it again dried for $12 \mathrm{~h}$ at $80^{\circ} \mathrm{C}$ in a vacuum oven [15].

Finally treated MWCNTs ( $0.5 \mathrm{~g}$ ) and GMA (35 g) were mixed in the $100 \mathrm{~mL}$ beaker with the homogenizer for $30 \mathrm{~min}$. The suspended substances were poured into flask. The flask was located in an oil bath at $120^{\circ} \mathrm{C} .3 \mathrm{mg}$ of 3-chlorotoluene as a catalyst was added into the flask, and the mixture was stirred for $3 \mathrm{~h}$ at $120^{\circ} \mathrm{C}$. And then, the GMA-MWCNT mixture was diluted with $\mathrm{CHCl}_{3}$ and vacuum filtered. The filtered GMAMWCNTs were thoroughly washed with $\mathrm{CHCl}_{3}$, and then filtered and dried overnight under vacuum at $80^{\circ} \mathrm{C}$ [16]. The scheme for GMA-MWCNTs was presented in Fig. 1.

Typically, the GMA-MWCNTs/PP nanocomposites were prepared by melt mixing in bravender $(30 \mathrm{rpm})$ at $200^{\circ} \mathrm{C}$ for $1 \mathrm{~h}$. To prepare samples for measuring the viscoelastic behaviors, finally obtained GMA-MWCNTs/PP nanocomposites were transformed to film form which has thickness of $1 \mathrm{~mm}$ and diameter of $25 \mathrm{~mm}$ by using hot press [17].

\subsection{Measurements and characterization}

The structural properties of pristine MWCNTs and GMAMWCNTs were investigated by FT-Raman measurements (RFS 100/S: Bruker, FT-Raman). The amount of grafted GMA was calculated by thermogravimetric analysis (STA 409 PC: NETZSCH, TGA). And the FT-IR (FT/IR-6100: JASCO) was used for the investigation of GMA-MWCNTs. The dispersion of GMA-MWCNTs in PP was analyzied using SEM (S-4200: HITACHI). And the viscoelastic properties were carried out using rheometer system (AR 2000ex: TA Instruments, Ltd, Rheometer) by frequency sweep procedure from 0.1 to $100 \mathrm{rad} /$ $\mathrm{s}$ and using period of shear rate from 0.1 to $10 \mathrm{~s}^{-1}$ at $200^{\circ} \mathrm{C}$.

\section{Results and Discussion}

FT-Raman was used to investigate surface features of

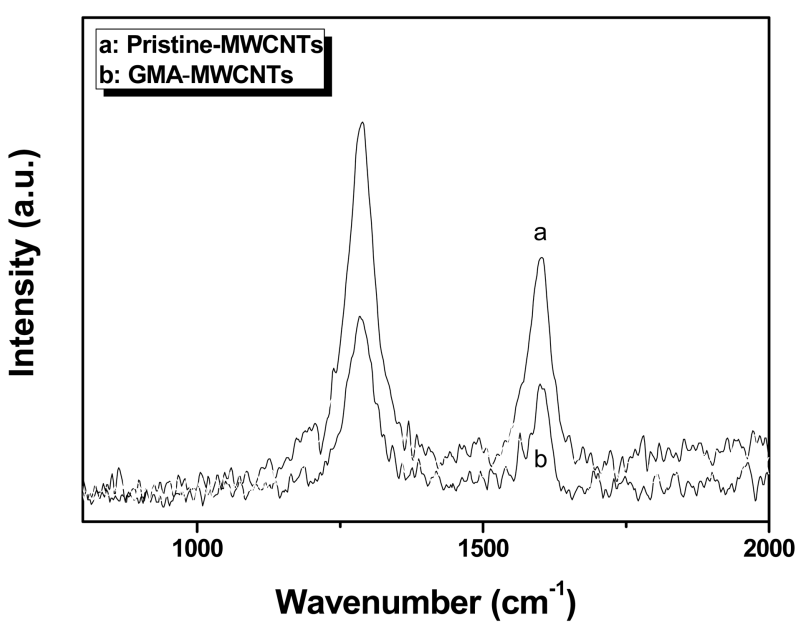

Fig. 2. Raman spectra of pristine MWCNTs and GMAMWCNTs.

GMA-MWCNTs. The Raman spectra normally use to carry out surface characteristics and structural properties of carbon based materials. Fig. 2 describes D band (defect or disorder mode) and $\mathrm{G}$ band (graphite or tangential mode) of FTRaman spectra for MWCNTs and GMA-MWCNTs. The D band is describing defect elements of MWCNTs. It was found regularly in the range of $1330 \sim 1360 \mathrm{~cm}^{-1}$. On the other hand, the $\mathrm{G}$ band meant flexible mode which was appeared from graphitic plane and matched with tangential shear of carbon atom. The $\mathrm{G}$ band was shown at $1580 \mathrm{~cm}^{-1}$ [16].

For all regions in the graph, the peak placements of $D$ band and $G$ band were not significantly changed after GMA grafting, but the peak intensity and area were slightly decreased. In this situation, it was considered that GMA was grafted on the surface of MWCNTs. In the intensity ratio of $\mathrm{D}$ band and $\mathrm{G}$ band $\left(\mathrm{I}_{\mathrm{D}} / \mathrm{I}_{\mathrm{G}}\right.$ ratio), $\mathrm{I}_{\mathrm{D}} / \mathrm{I}_{\mathrm{G}}$ value (1.64) of GMAMWCNTs was higher than that of pristine MWCNTs (1.56). It was considered that relative increase of $\mathrm{I}_{\mathrm{D}} / \mathrm{I}_{\mathrm{G}}$ value was caused by partly increment of structural amorphous surface of GMA-MWCNTs [17,18].

To calculate GMA mass which was grafted on the surface of MWCNTs, TGA was used. Prepared pristine MWCNTs and GMA-MWCNTs were measured from room temperature to $800^{\circ} \mathrm{C}$ in $\mathrm{N}_{2}$ atmosphere. As shown in Fig. 3, pristine MWCNTs showed $15 \mathrm{wt} \%$ decreases. On the other hand, GMA-MWCNTs was remarked about $45 \mathrm{wt} \%$ mass loss started from $200^{\circ} \mathrm{C}$, continued rapidly nearby $300^{\circ} \mathrm{C}$ and after $650^{\circ} \mathrm{C}$ it was remained about $55 \mathrm{wt} \%$. For these reasons, it was concluded the GMA was grafted with 45 $\mathrm{wt} \%$ mass ratio on the MWCNT surfaces [19].

Fig. 4 shows the FT-IR spectra of (a) pristine MWCNTs and (b) GMA-MWCNTs. In pristine MWCNTs, there are no measurable functional groups. On the other hand, after functionalization, GMA-MWCNTs show a band at $3400 \mathrm{~cm}^{-1}$ which is attributed to the $-\mathrm{OH}$ stretching vibrations by the 


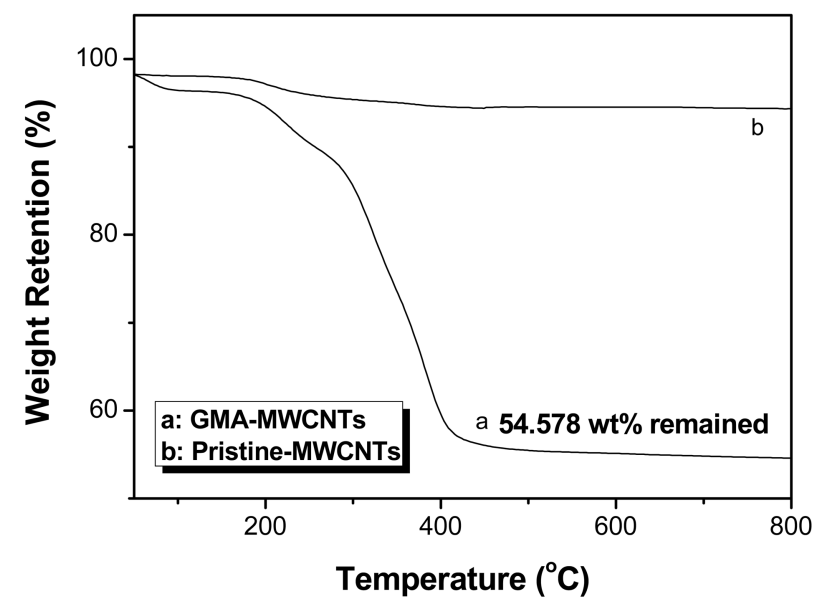

Fig. 3. TGA results of (a) pristine MWCNTs and (b) GMAMWCNTs.

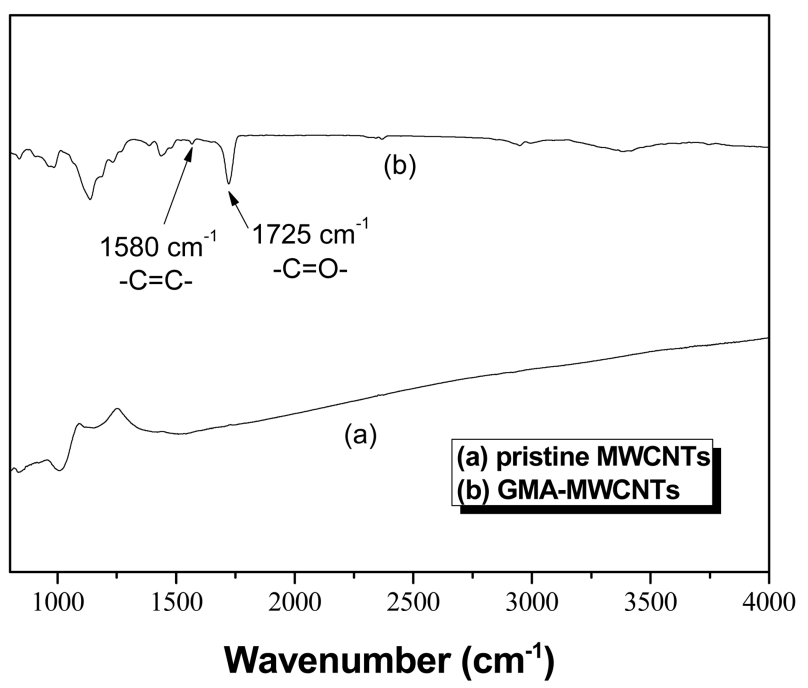

Fig. 4. FT-IR spectra of (a) pristine MWCNTs and (b) GMAMWCNTs.

grafting of GMA onto MWCNTs. And a band of $1725 \mathrm{~cm}^{-1}$ indicates to the $-\mathrm{C}=\mathrm{O}$ stretching vibration of $\mathrm{GMA}$ MWCNTs. In addition, GMA-MWCNTs exhibits a band at $1580 \mathrm{~cm}^{-1}$ attributed to $\mathrm{C}=\mathrm{C}$ double bond. From above results, GMA had covalently bonded on the surface of MWCNTs through ring-opening reaction [16].

Fig. 5 shows the SEM images of GMA-MWCNTs/PP nanocomposites. From Fig. 5, white parts which are indicated by white arrows were GMA-MWCNTs embed in PP matrix. It can be seen that the GMA-MWCNTs were uniformly dispersed in the PP matrix without aggregation in the case of both low $(0.5 \mathrm{wt} \%)$ and high $(2.0 \mathrm{wt} \%)$ GMAMWCNTs contents. This is due to the empowered compatibility between MWCNTs and PP matrix by GMAgrafting [20].

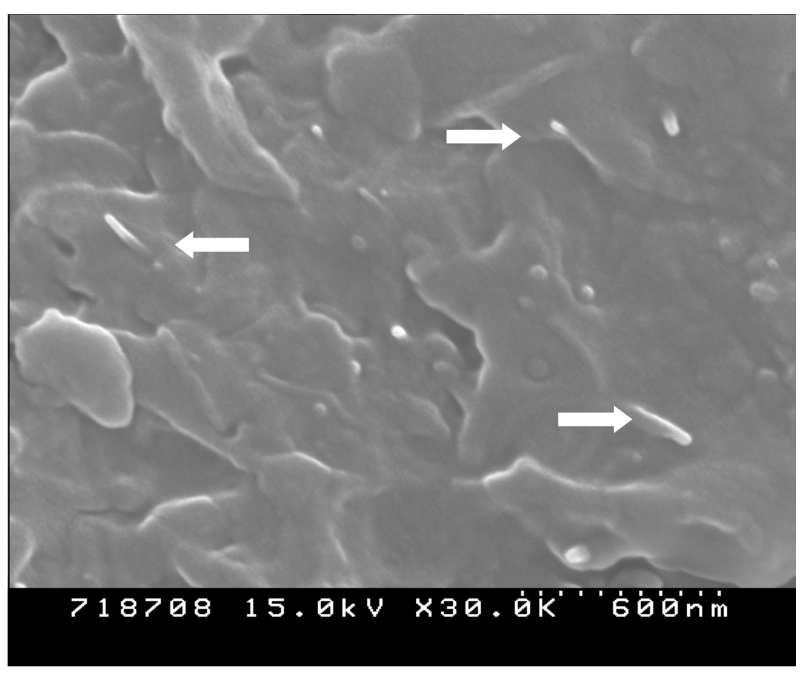

(a)

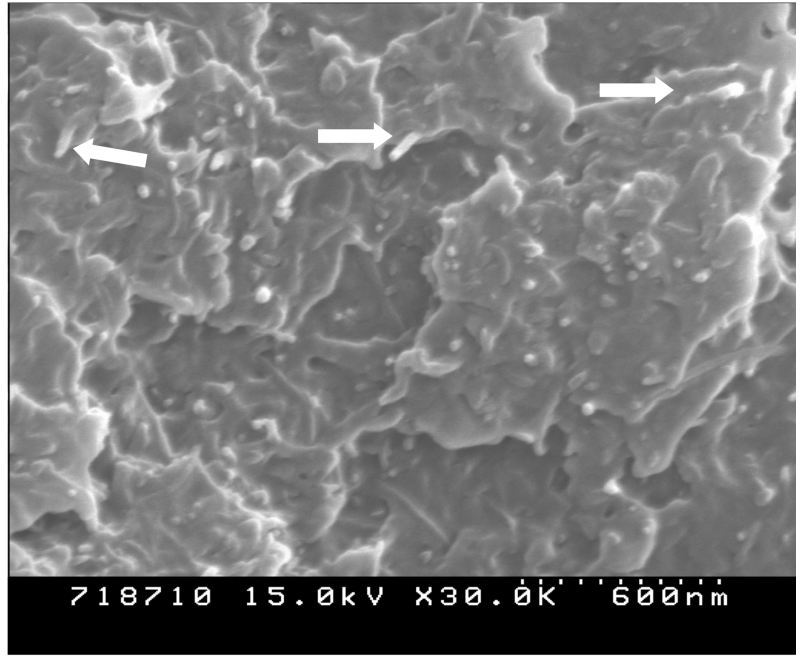

(b)

Fig. 5. SEM images of (a) GMA-MWCNTs 0.5 and (b) GMAMWCNTs 2.0.

The viscoelastic behaviors of PP matrix are from intramolecular forces which were caused by polymer chain's positioning in physicochemical bonding vectors and changes of strain from transformation of liquid state. In this study, the effect of GMA-MWCNTs on viscoelastic behaviors of PP matrix was investigated. It can be seen that the addition of GMA-MWCNTs caused viscoelastic changes of GMAMWCNTs/PP nanocomposites. Additionally, the enhancement of interfacial interaction between GMA-MWCNTs and PP matrix could be affected to viscoelastic behaviors of GMAMWCNTs/PP nanocomposites.

The results of the shear viscosity by measuring the steady share rate for the GMA-MWCNTs/PP nanocomposites are shown in Fig. 6. Because of intermolecular interaction between GMA-MWCNTs and PP matrix, it could be 


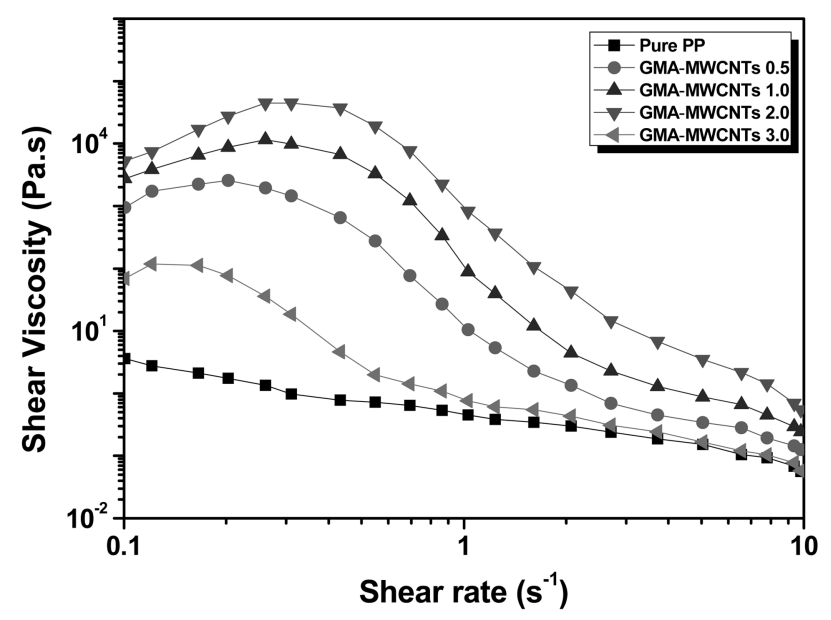

Fig. 6. The shear viscosity of GMA-MWCNTs/PP nanocomposites with GMA-MWCNT content.
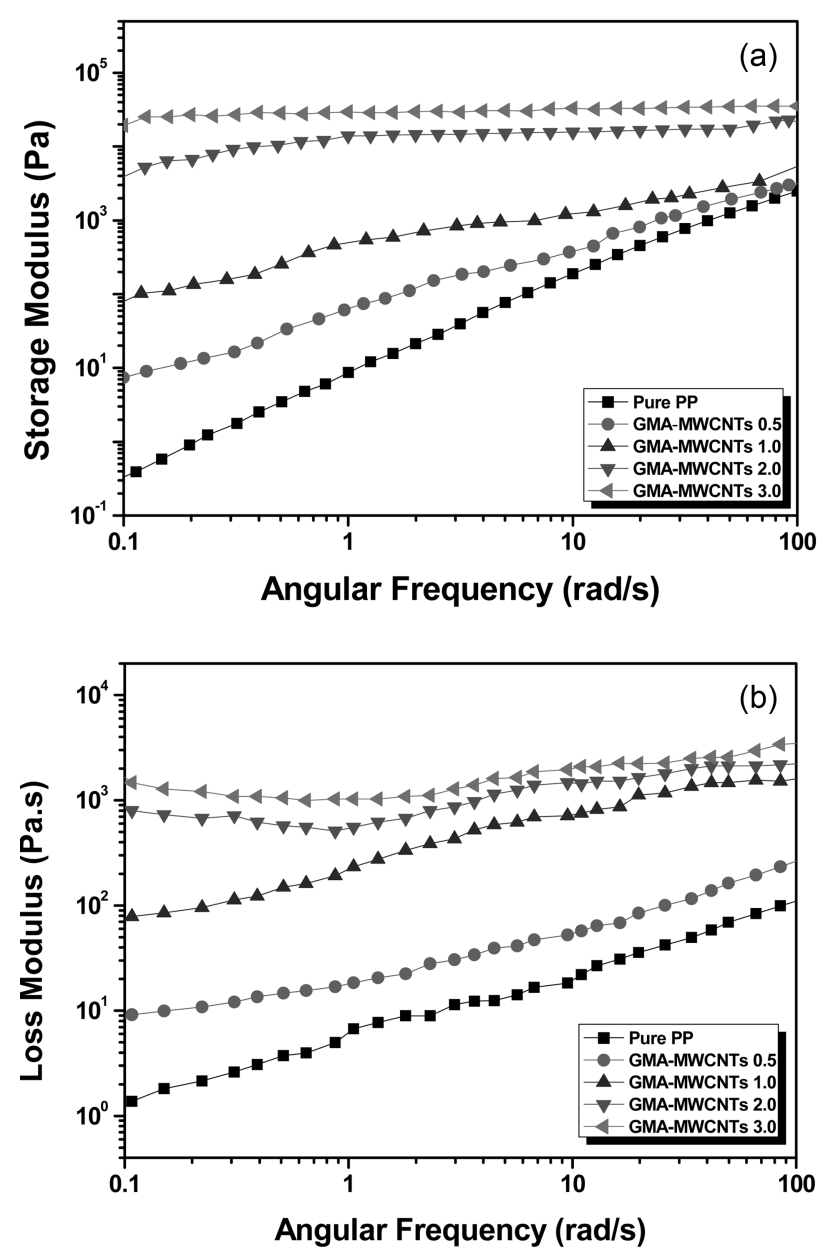

Fig. 7. The storage modulus (a) and loss modulus (b) of GMAMWCNTs/PP nanocomposites with GMA-MWCNT content.

predicted that the shear viscosity increased with increasing the GMA-MWCNTs content. As a result in Fig. 6, at the low frequencies, the shear viscosity of the GMA-MWCNTs/PP nanocomposites clearly deviated from the liquid-like behavior of the neat matrix with increasing the GMAMWCNTs content. In other words, the pure PP exhibits the liquid-like behaviors at all frequency region and the viscosity of the PP increases as the GMA-MWCNTs increases. With addition of GMA-MWCNTs, the GMAMWCNTs/PP nanocomposites show the non Newtonian behavior at low shear rate. It is attributed to the strong interaction between MWCNT-MWCNT, leading to non Newtonian behaviors of GMA-MWCNTs/PP nanocomposites at low shear rate [21]. With frequency rising, the shear viscosity of the GMA-MWCNTs/PP nanocomposites became Newtonian behavior. But in case of GMA-MWCNTs 3.0 sample, the shear viscosity had the lowest values in the GMA-MWCNTs/PP nanocomposites. It was seen that the excess addition of GMA-MWCNTs had influenced to decrease the shear viscosity, resulting from the aggregation of GMA-MWCNTs.

The changes of storage modulus $\left(\mathrm{G}^{\prime}\right)$ and loss modulus $\left(\mathrm{G}^{\prime \prime}\right)$, at different GMA-MWCNTs contents in PP matrix are shown in Fig. 7 (a) and (b). It could be found that $\mathrm{G}^{\prime}$ and $\mathrm{G}^{\prime \prime}$ of PP matrix seem to reach the plateaus at low frequencies with relatively high GMA-MWCNTs content of $2.0 \mathrm{wt} \%$ and $3.0 \mathrm{wt} \%$.

Fig. 7 (a) shows changes of the storage modulus. At low frequency, $\mathrm{G}^{\prime}$ was increased with increasing GMAMWCNTs content and frequency. In high frequency region, the increase of $\mathrm{G}^{\prime}$ was reduced. Also, in Fig. 7 (b), loss modulus shows similar behaviors like storage modulus at all frequency region. It could be explained that the increase of GMA-MWCNTs content led to the formation of a CNT network and the transition from liquid-like to solid-like. These results provide the enhanced rheological properties by the improved interfacial interaction behavior between GMAMWCNTs and PP matrix [22].

\section{Conclusion}

In this work, we investigated the viscoelastic behaviors of glycidyl methacrylate grafted multi-walled carbon nanotubes (GMA-MWCNTs)/polypropylene (PP) nanocomposites. The surface features of GMA-MWCNTs and the viscoelastic behaviors of GMA-MWCNTs/PP nanocomposites were investigated. As a result, rheological properties, such as storage modulus and loss modulus of neat PP were enhanced with increasing GMA-MWCNTs content. The shear viscosity was also increased with increasing GMA-MWCNTs content. Consequently, it was found that the chemical grafting of GMA on the MWCNT surfaces was affected to enhance the interfacial interaction between the MWCNTs and the PP matrix in this system. 


\section{References}

[1] Dai, L. M.; Mau, A. W. H. Adv. Mater. 2001, 13, 899.

[2] Baughman, R. H.; Zakhidov, A. A.; De Heer, W. A. Science 2002, 297, 787.

[3] Sun, Y. P.; Fu, K. F.; Lin, Y.; Huang, W. J. Acc. Chem. Res. 2002, 35, 1096.

[4] Dai, H. J. Surf. Sci. 2002, 500, 218.

[5] Modi, A.; Koratkar, N.; Lass, E.; Wei, B. Q.; Ajayan, P. M. Nature 2003, 424, 171.

[6] Ghosh, S.; Sood, A. K.; Kumar, N. Science 2003, 299, 1042.

[7] Tans, S. J.; Verschueren, A. R. M.; Dekker, C. Nature 1998, 393, 49.

[8] Postma, H. W. C.; Teepen, T.; Yao, Z.; Grifoni, M.; Dekker, C. Science 2001, 293, 76.

[9] Javey, A.; Kim, H.; Brink, M.; Wang, Q.; Ural, A.; Guo, J.; Mcintyre, P.; Mceuen, P.; Lundstrom, M.; Dai, H. J. Nat. Mater. 2002, 1, 241.

[10] Bachtold, A.; Hadley, P.; Nakanishi, T.; Dekker, C. Science 2001, 294, 1317.

[11] Sung, Y. T.; Han, M. S.; Song, K. H.; Jung, J. W.; Lee, H. S.; Kum, C. K.; Joo, J.; Kim, W. N. Polymer 2006, 47,
4434.

[12] Fischer, J. E.; Dai, H.; Thess, A.; Lee, R.; Hanjani, N. M.; Dehaas, D. L. Phys. Rev. B. 1997, 55, 4921.

[13] Zdenko, S.; Dimitrios, T.; Konstantinos, P.; Costas, G. Prog. Polym. Sci. 2010, 35, 357.

[14] Mohammed, H. A.; Uttandaramen, S. Carbon 2009, 47, 2.

[15] Hou, P. X.; Liu, C.; Cheng, H. M. Carbon 2008, 46, 2003.

[16] Zheng, Y.; Zhang, J.; Xidadong, Z.; Chen, W.; Wang, R. J. Appl. Polym. Sci. 2009, 112, 1755.

[17] Meyyappan, M. "Carbon Nanotubes Science and Applications", CRC press, Boca Raton, 2005, ???.

[18] Park, S. J. "Interfacial Forces and Fields: Theory and Application", ed. J. P. Hsu, Marcel Dekker, New York, 1999, Chap 9.

[19] Lee, Y. S.; Im, J. S.; Yun, S. M.; Nho, Y. C.; Kang, P. H.; Jin, H. K. Carbon Lett. 2009, 10, 314.

[20] Kim, K. S.; Choi, K. E.; Park, S. J. Carbon Lett. 2009, 10, 335

[21] Lee, S. H.; Kim, M. W.; Kim, S. H.; Youn, J. R. Europ. Polym. J. 2008, 44, 1620.

[22] Seo, M. K.; Lee, J. R.; Park, S. J. J. Mater. Sci. Eng. A. 2005, 404, 79 . 